\title{
Protein co-migration database (PCoM -DB) for Arabidopsis thylakoids and Synechocystis cells
}

\author{
Atsushi Takabayashi ${ }^{1,2^{*}}$, Ryosuke Kadoya ${ }^{1}$, Masayoshi Kuwano ${ }^{1}$, Katsunori Kurihara', Hisashi Ito ${ }^{1,2}$, \\ Ryouichi Tanaka ${ }^{1,2}$ and Ayumi Tanaka, ${ }^{1,2}$
}

\begin{abstract}
Protein-protein interactions are critical for most cellular processes; however, many remain to be identified. Here, to comprehensively identify protein complexes in photosynthetic organisms, we applied the recently developed approach of blue native PAGE (BN-PAGE) coupled with LC-MS/MS to the thylakoid proteins of Arabidopsis thaliana and the whole cell proteins of whole cell proteins of Synechocystis sp. PCC 6803. We identified 245 proteins from the purified Arabidopsis thylakoid membranes and 1,458 proteins from the whole cells of Synechocystis using the method. Next, we generated protein migration profiles that were assessed by plotting the label-free estimations of protein abundances versus migration distance in BN-PAGE. Comparisons between the migration profiles of the major photosynthetic complexes and their band patterns showed that the protein migration profiles were well correlated. Thus, the protein migration profiles allowed us to estimate the molecular size of each protein complex and to identify co-migrated proteins with the proteins of interest by determining the protein pairs that contained peaks in the same gel slice. Finally, we built the protein co-migration database for photosynthetic organisms (PCoM-DB: http://pcomdb.lowtem.hokudai.ac.jp/proteins/top) to make our data publicly accessible online, which stores the analyzed data with a user-friendly interface to compare the migration profiles of proteins of interest. It helps users to find unidentified protein complexes in Arabidopsis thylakoids and Synechocystis cells. The accumulation of the data from the BN-PAGE coupled with LC-MS/MS should reveal unidentified protein complexes and should aid in understanding the adaptation and the evolution of photosynthetic organisms.
\end{abstract}

Keywords: Protein complex, Proteome, Arabidopsis thaliana, Synechocystis sp. PCC 6803, Database

\section{Background}

Cellular processes are not only performed by the coordinated activities of individual proteins, but they are also executed by a wide variety of protein complexes. Consequently, the identification of protein complexes is important for understanding protein structure and function and also for understanding dynamic cellular processes. For photosynthetic organisms, photosynthetic cells are where photosynthesis and many other metabolic pathways are highly inter-connected via metabolic products. In addition, light harvesting systems of photosynthetic organisms, which are complexes of pigments and proteins, have been dynamically

\footnotetext{
* Correspondence: takabayashi@pop.lowtem.hokudai.ac.jp
'Institute of Low Temperature Science, Hokkaido University, N19 W8 Kita-Ku,

* Correspondence: takabayashi@pop.lowtem.hokudai.ac.jp
${ }^{1}$ Institute of Low Temperature Science, Hokkaido University, N19 w8 Kita-Ku, Sapporo 060-0819, Japan

${ }^{2}$ Japan Core Research for Evolutionary Science and Technology (CREST), Sapporo, Japan
}

\section{Springer}

changed during evolution (Neilson and Durnford 2010). Even among cyanobacteria, the light harvesting systems of Prochlorococcus are significantly different from other cyanobacteria (Wiethaus et al. 2010). Therefore, a comprehensive identification of the protein complexes in photosynthetic cells is critical for understanding the regulation of photosynthesis and other metabolic processes.

The number of sequenced photosynthetic organisms has been growing rapidly with advances in DNA sequencing technologies. The availability of whole genomic sequences and the development of mass spectrometry-based identification techniques have enabled us comprehensive protein identification in many photosynthetic organisms. For example, the large-scale identification of proteinprotein interactions has achieved significant results using a model plant Arabidopsis thaliana (e.g. (Arabidopsis Interactome Mapping Consortium 2011) and see also a recent review by Braun et al. (Braun et al. 2013)). In another 
example, for cyanobacteria, a protein-protein interaction database of a model cyanobacteria Synechocystis sp. PCC 6803 is also constructed (Kim et al. 2008). However, the methods for large-scale identification of protein-protein interactions are still labor-intensive and time-consuming. Therefore, a rapid, sensitive, less laborious, and comprehensive approach for identifying protein complexes is necessary to obtain a better understanding of cellular processes in growing number of sequenced photosynthetic organisms.

Blue native PAGE (BN-PAGE) is developed for the high-resolution separation method of protein complexes in their structurally and enzymatically intact forms (Schägger and von Jagow 1991). The method has been shown as an useful method to separate protein complexes, especially for large, labile, and membrane protein complexes (Wittig and Schägger 2008). The general workflow for identification of protein complexes comprises the separation of protein complexes by onedimensional (1D) BN-PAGE followed by a denaturing second-dimensional (2D) electrophoresis by SDS-PAGE, in which each protein complex dissociate into each individual protein. Then, stained protein spots with $\mathrm{CBB}$, silver, or fluorescence dyes were identified by LC-MS/MS. Alternatively, the modified method of BN-PAGE coupled with LC-MS/MS has recently been developed. Fandino et al. firstly demonstrated that the BN-PAGE directly coupled with LC-MS/MS approach can identify the membrane protein complexes (Fandino et al. 2005). Helbig et al. and Wessels et al. extended the method to cover the whole length of 1D BN-lanes for LC-MS/MS (Helbig et al. 2009; Wessels et al. 2009). They demonstrated that coupling of the method with protein migration/correlation profiling is useful for finding potentially interacting proteins using mitochondrial membranes isolated from the yeast (Helbig et al. 2009) and the human embryonic kidney 293 (Wessels et al. 2009). The application of the approach to the tobacco BY-2 cells (Remmerie et al. 2011) and rat hart mitochondria (Heide et al. 2012) has been also reported.

In the first step of the method, protein complexes are solubilized by a mild detergent, such as dodecyl maltoside, and separated by BN-PAGE. Second, the BN gel is horizontally cut into slices, and the proteins in each BN gel slice are in-gel digested with trypsin and identified by LCMS/MS. Then, the similarities between the protein migration profiles across gel slices, which are generated based on estimations of the protein abundances using label-free semi-quantitative methods, can be used to estimate potential protein-protein interactions. Comparing to the standard 2D-BN/SDS-PAGE coupled with LC-MS/MS, the omission of the second step SDS-PAGE has some advantages. The principle improvement of the method is that the rapid and simple approach from BN-gels to LC-MS /MS allows us the analysis of protein complexes without labor-intensive and time-consuming step, which is suitable for semi high-throughput analysis. In addition, the $\mathrm{BN}$ PAGE coupled with LC-MS/MS does not suffer incomplete spot detection due to the limited dynamic range of the staining method (Remmerie et al. 2011). Furthermore, artifactual methionine oxidation by 2D-SDS-PAGE is prevented (Fandino et al. 2005).

In this study, we applied the BN-PAGE coupled with LC-MS/MS method to Arabidopsis thylakoid membranes and the whole cells of cyanobacterium Synechocystis sp. PCC 6803, model organisms widely used in photosynthesis studies, to systematically identify protein complexes and to construct a comprehensive and a user-friendly database. The web database helps users obtain information on selecting protein complexes of interest and to identify unknown protein complexes in Arabidopsis thylakoid membranes and in cells of Synechocystis sp. PCC 6803.

\section{Results}

Identification of isolated Arabidopsis thylakoid membrane proteins

We purified the Arabidopsis thylakoid membranes using sucrose density gradients. The membranes were then solubilized with dodecyl maltoside and separated by BNPAGE. The band pattern of the separated thylakoid protein complexes in the CBB staining gel was essentially the same as the profile of WT identified in our previous report (Takabayashi et al. 2011). Based on the identifications in that report, the bands of the four PSII-LHCII supercomplexes, the dimeric PSII and the PSI-LHCI supercomplex, the PSII monomer, the LHCII assembly (the CP29-CP24-LHCII trimer), and the trimeric LHCII visualized by $\mathrm{CBB}$ staining were identified (Figure 1).

To identify the thylakoid proteins separated by $\mathrm{BN}$ PAGE, we sliced a BN gel lane horizontally into 60 pieces from the top of the gel to immediately before the dye front (Figure 1). These gel slices were processed for in-gel digestion with trypsin and analyzed by LC-MS/MS. In total, 245 proteins were identified from the 60 gel slices (Additional file 1: Table S1). The purified thylakoid membranes may have been contaminated by other cellular fractions. To estimate the number of thylakoid-localized proteins among all the identified proteins, we compared our results with the recent large-scale thylakoid proteomic study by Ferro et al. (Ferro et al. 2010). According to their results, 102 of the 245 proteins were classified as thylakoid proteins, and 33 proteins were classified as envelope or stromal proteins (Additional file 1: Table S1). In addition, we identified 15 known thylakoid-localized proteins that were not classified as thylakoid proteins by Ferro et al. (Ferro et al. 2010), including NDH proteins (At1g14150, At1g70760, At2g39470, At5g43750, At5g58260, AtCg00420, AtCg00430, AtCg01010, AtCg01090), Lil3 proteins (At4g17600, At5g47110), Lhca5 (At1g45474), LPA3 


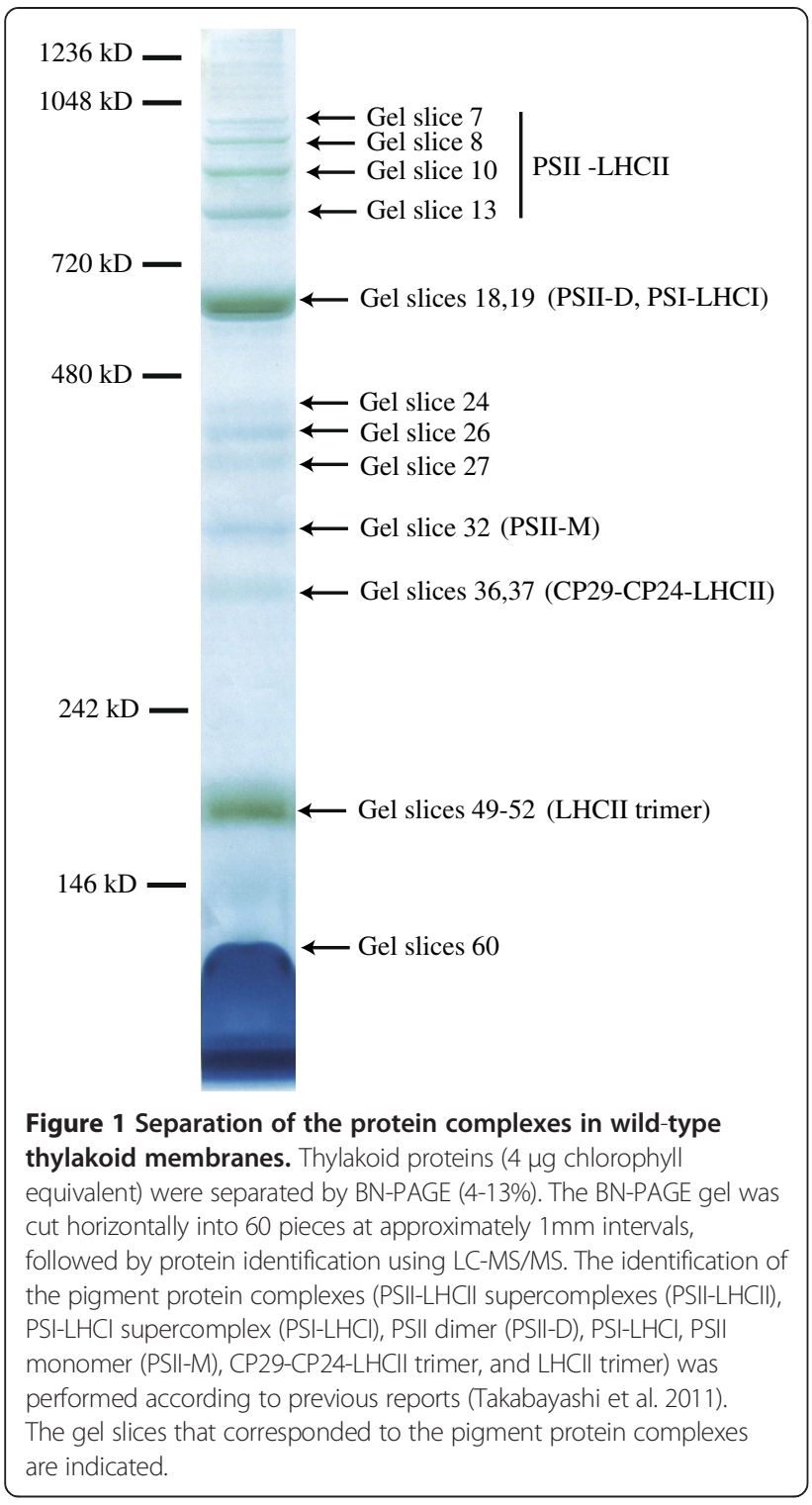

(At1g73060), STN7 (At1g68830), and YCF4 (AtCg00520). Therefore, a minimum of 117 of the 245 proteins (48\%) were likely thylakoid-localized proteins, whereas the functions of the remaining 95 proteins were largely unknown.

To verify the enrichment of the thylakoid proteins in our protein sample, we estimated the abundances of the identified proteins in the protein samples using the emPAI (exponentially modified protein abundance index) method (Ishihama et al. 2005). The emPAI method, which is based on the numbers of observed peptides per protein by LC-MS/MS and is further normalized by the observable number of peptides, has been used for label-free semiquantitative estimates of relative protein abundances and has been reported to exhibit a good correlation with protein abundance in samples (Ishihama et al. 2005).
According to the paper, we calculated the protein content in molar percentages using the emPAI values (Additional file 1: Table S1) and found that the 117 putative thylakoid proteins in our results accounted for $97 \%$ of all the identified proteins, suggesting that the thylakoid proteins were substantially enriched in the sample.

\section{The emPAI-based profiles of the subunits in the thylakoid} large protein complexes were well correlated with their separation patterns in BN-PAGE

The protein migration profiles, which were assessed by the label-free estimation of protein abundances versus the migration distance in BN-PAGE, were reported to be substantially similar among subunits in the same protein complexes (Helbig et al. 2009; Wessels et al. 2009; Remmerie et al. 2011). Label-free quantitative approaches are inexpensive and have a considerable analytical depth and dynamic range, although quantitative precision is typically lower compared to stable-isotope approaches (Schulze and Usadel 2010).

In this study, we generated the emPAI-based protein migration profiles across all the $\mathrm{BN}$ gel slices, in which each emPAI value was plotted on the y-axis, and each gel slice number (ordered from top to bottom) was plotted on the $\mathrm{x}$-axis, based on the Mascot data from each gel slice (Additional file 2: Table S2). Before using the emPAI-based protein migration profiles for identifying protein complexes, we first verified the correlation between the emPAI-based protein migration profiles and their band patterns by comparing the emPAI-based migration profiles of the PSII proteins with their band patterns, which were visualized by the CBB staining on the BN-PAGE.

The bands of the PSII-LHCII supercomplexes, the PSII dimer, and the PSII monomer were visualized by the CBB staining in the region on the BN-PAGE gel lane corresponding to the slices between 7 and 13, slices 18 and 19, and slice 32, respectively (Figure 1). Meanwhile, the emPAI-based migration profiles of four PSII core subunits (D1 (PsbA), CP47 (PsbB), CP43 (PsbC), and D2 $(\mathrm{PsbD}))$ had peaks that corresponded to the bands of the PSII complexes from the BN-PAGE (Figure 2A). The emPAI-based migration profiles of the remaining PSII core proteins (PsbE, PsbH, and PsbL) were also similar (Additional file 3: Figure S1). Likewise, the emPAIbased migration profiles of LHCII proteins had peaks that corresponded to the PSII-LHCII supercomplex (the slices between 7 and 13), the CP29-CP24-LHCII supercomplex (slices 36 and 37), and the LHCII trimer (the slices between 49 and 52) in the BN-PAGE, respectively (Figure 2B). The emPAI values in the peaks of PsbA and PsbD were much smaller than those of PsbC and PsbC (Figure 2A), likely because their hydrophobicity. The likelihood of the MS-based protein detection varied 


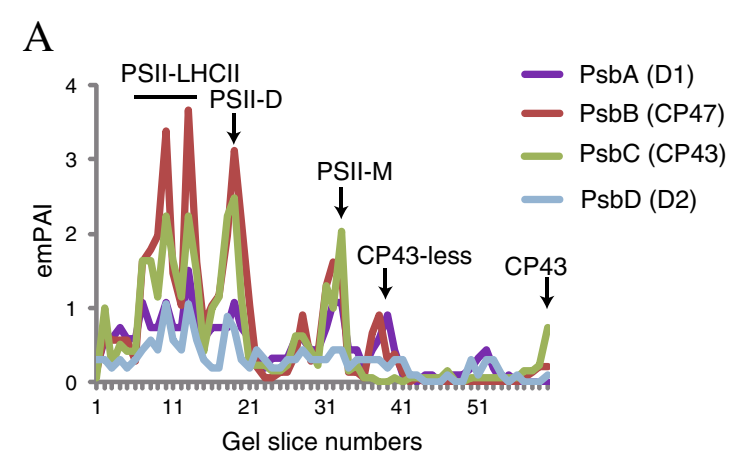

B

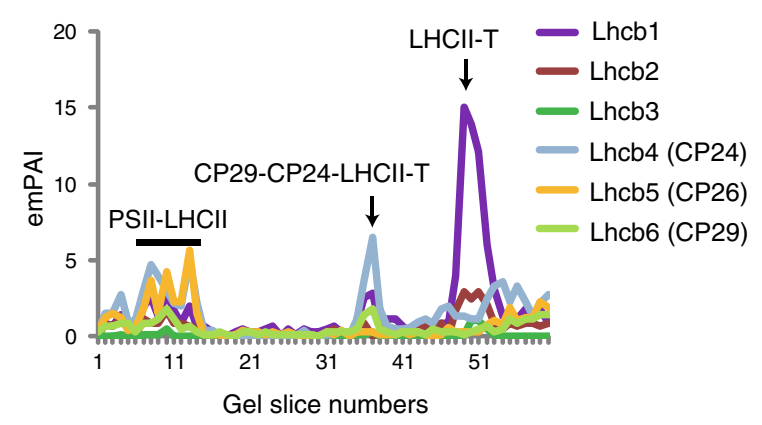

$\mathrm{C}$
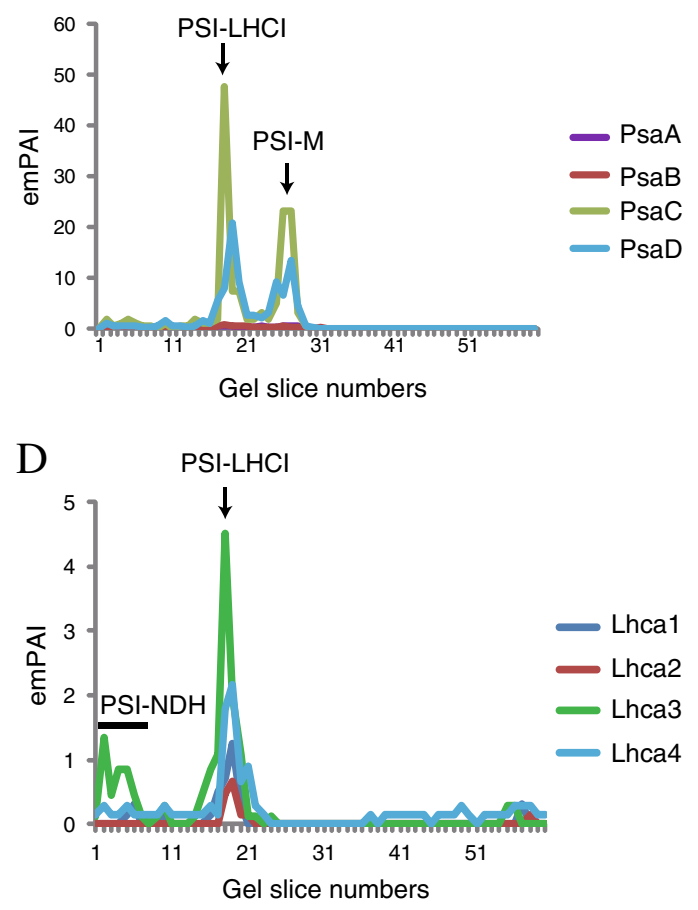

Figure 2 Protein migration profiles of the PSII, LHCII, PSI, and LHCI proteins. The emPAl-based protein migration profiles of the PSII core subunits (PsbA, PsbB, PsbC, and PsbD) (A), LHCll proteins (Lhcb1-6) (B), four PSI core subunits (PsaA, PsaB, PsaC, and PsaD) (C), and $\mathrm{LHCl}$ proteins (Lhca1-4) (D) are shown. The emPAl value of PsaD was the sum of the emPAl values of PsaD1 and PsaD2. The pigment protein complexes corresponding to the peaks in the migration profiles of the PSI-NDH supercomplex (PSI-NDH), PSII-LHCII supercomplex (PSII-LHCII), PSI-LHCI supercomplex (PSI-LHCI), PSII dimer (PSII-D), PSI-LHCI, PSII monomer (PSII-M), CP29-CP24-LHCII trimer, and LHCII trimer) are indicated.

greatly depending on the properties of each peptide, such as the peptide length, net charge, and solubility (Lu et al. 2007). Especially, the probability of identifying very hydrophobic proteins is typically low.

In addition to PSII, the PSI-LHCI supercomplex (the slices between 17 and 20) and the PSI monomer (the slices between 26 and 28) were visualized in BN-PAGE by the $\mathrm{CBB}$ staining (Figure 1), and the emPAI-based migration profiles of four PSI core proteins (PsaA, PsaB, PsaC, and PsaD) had peaks that corresponded to the CBB-stained bands of the PSI-LHCI supercomplex and the PSI monomer (Figure 2C). The emPAI values of $\mathrm{PsaA} / \mathrm{PsaB}$ were much smaller than those of $\mathrm{PsaC}$ and PsaD, likely because their hydrophobicity. All of the remaining PSI proteins, except for PsaN, exhibited similar emPAI-based migration profiles (Additional file 3: Figure S1). The emPAI-based migration profiles of the LHCI proteins also contained major peaks corresponding to the PSI-LHCI supercomplex (Figure 2D).

Although the large thylakoid protein complexes, cytochrome $b_{6} / f$ complex, ATP synthase, and the NDH complex, were not clearly visualized in the BN gel (Figure 1), we previously reported their oligomeric states in the $\mathrm{BN}$ gel using 2D-BN/SDS-PAGE (Takabayashi et al. 2011). Consequently, we further verified whether the emPAI-based migration profiles of the subunits in the large thylakoid protein complexes were consistent with their respective oligomeric states.

The emPAI-based migration profiles of the four proteins of cytochrome $b_{6} / f$ complex (PetA, PetB, PetC, and PetD) contained a peak that likely corresponded to the dimeric form of the complex at gel slice 32 (Figure 3A), and the emPAI-based migration profiles of the chloroplastic ATP synthase proteins (AtpA, AtpB, AtpC1, AtpD, AtpE, AtpF, AtpI, and AT4G32260) had a peak that corresponded to the $\mathrm{CF}_{0} \mathrm{~F}_{1}$ complex at gel slice 19 and some of them (AtpA, AtpB, AtpC1, and AtpE) also had a peak that corresponded to the $\mathrm{CF}_{1}$ subunit at gel slice 28 (Figure 3B and Additional file 3: Figure S1).

In addition to the major protein complexes of the thylakoid membranes, $15 \mathrm{NDH}$ proteins were identified in this study (Additional file 1: Table S1). The nomenclature of NDH proteins were followed by Ifuku et al. (2011). The 

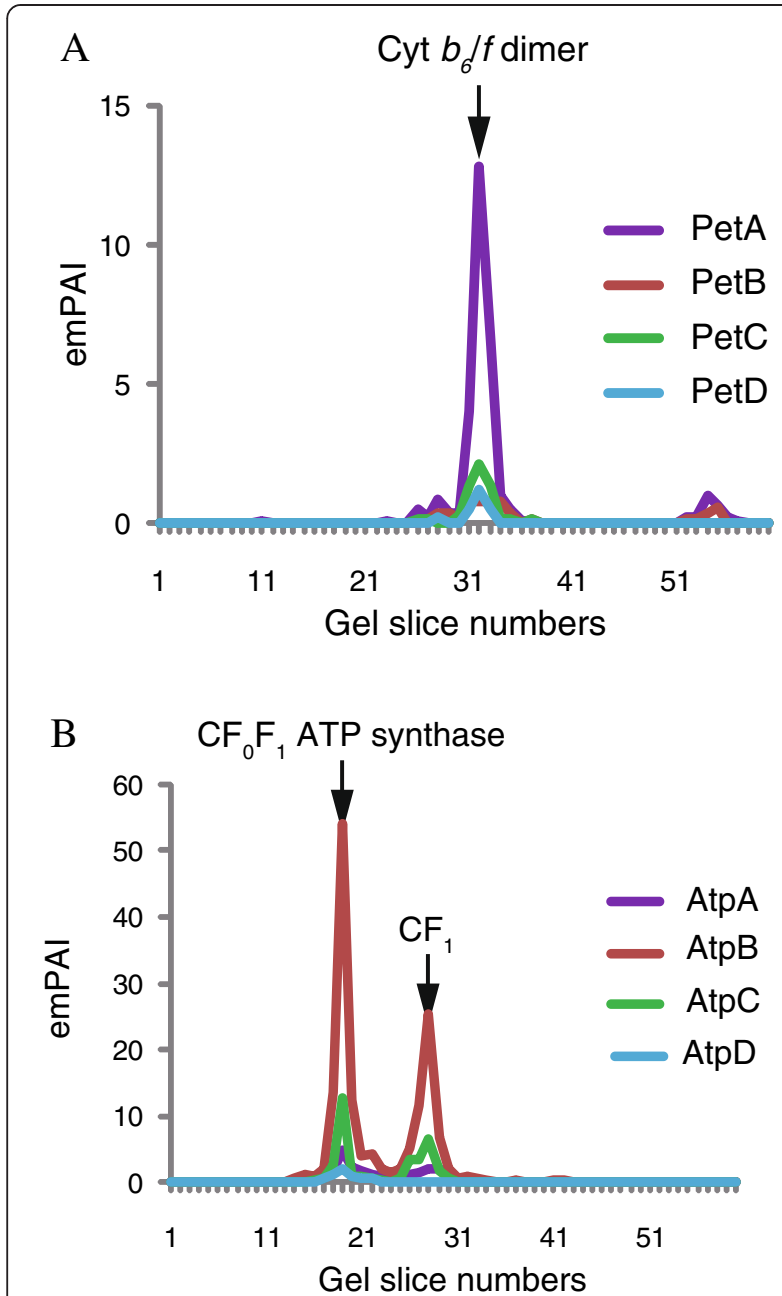

$\mathrm{C}$

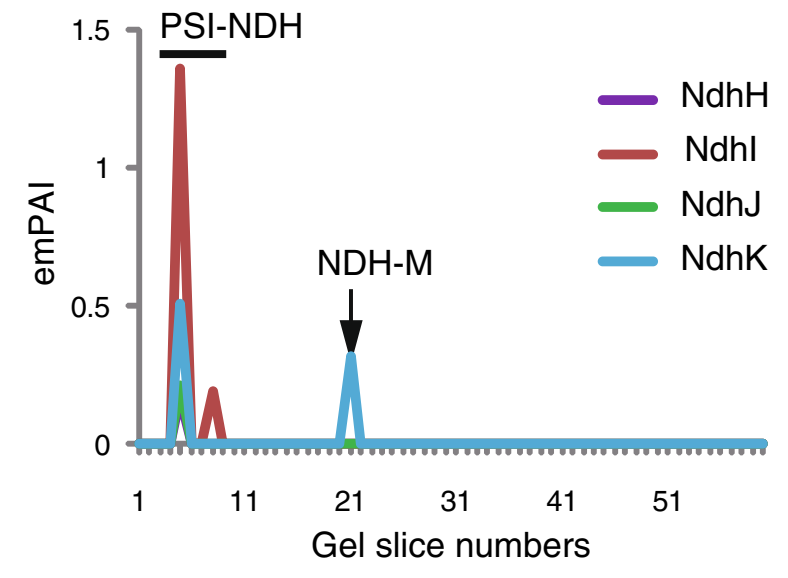

Figure 3 Protein migration profiles of four representative subunits from the cytochrome $b_{6} / f$, ATP synthase, and NDH proteins. The emPAI-based protein migration profiles of the cytochrome $b_{6} / f$ proteins (PetA, PetB, PetC, and PetD) (A), ATP synthase proteins (AtpA, AtpB, AtpC, and AtpD) (B), and NDH proteins ( $\mathrm{NdhH}, \mathrm{Ndhl}, \mathrm{NdhJ}$, and $\mathrm{NdhK}$ ) (C) are shown. The protein complexes corresponding to the peaks in the migration profiles of the cytochrome $b_{6} / f$ dimer, CFoF 1 ATP synthase, $\mathrm{CF}_{1}$ subcomplex, PSI-NDH supercomplex (PSI-NDH), and NDH monomer (NDH) were estimated based on the oligomeric states of the protein complexes. For the NDH proteins, we followed the nomenclature proposed by Ifuku et al. (2011) (Ifuku et al. 2011).

major form of the NDH complex in Arabidopsis is the PSI-LHCI supercomplex, which consists of the PSI-LHCI and the monomeric NDH, with Lhca5 and Lhca6 proteins acting as linker proteins (Ifuku et al. 2011). The emPAIbased migration profiles of all the identified NDH subunits, except PnsL5, contained a peak in slice 5 (above the bands of the PSII supercomplexes) (Figure $3 \mathrm{C}$ and Additional file 4: Figure S2) that corresponded to the PSINDH supercomplex. Many of the PSI and LHCI proteins were also identified in slice 5. In addition, PnsB1, PnsB2, PnsB5, and PnsL3 proteins were also identified in the slices between 11 and 13 (Figure 3C and Additional file 4: Figure S2), likely corresponding to the monomeric NDH complex.

These results demonstrated that the subunits from the same protein complex generally exhibited similar emPAIbased migration profiles. In addition, the emPAI-based protein migration profiles can be used to reveal the oligomeric states of the photosynthetic protein complexes and used to find the co-migrated proteins with a protein of interest.

However, it should be noted that some of the PSI and PSII subunits were not identified in this study. Since the probability of identifying a small protein and a very insoluble protein were typically low, the small subunits of the PSI and the PSII may be difficult to identify by LC-MS/MS. Future technical developments should be required to comprehensive identify protein complexes.

\section{Analysis of the whole cell proteins of Synechocystis sp. PCC 6803}

Using Arabidopsis thylakoid membranes as a model, our data presented here showed that BN-PAGE coupled with LC-MS/MS was useful for identifying potential protein complexes. One of the goals of our research is the comprehensive identification of protein complexes in the whole cells of photosynthetic organisms to aid in understanding their cellular processes. Therefore, we applied the described method to the whole cell proteins of the cyanobacterium Synechocystis sp. PCC 6803.

The whole cells of Synechocystis sp. PCC 6803 were homogenized using glass beads on a vortex mixer. 


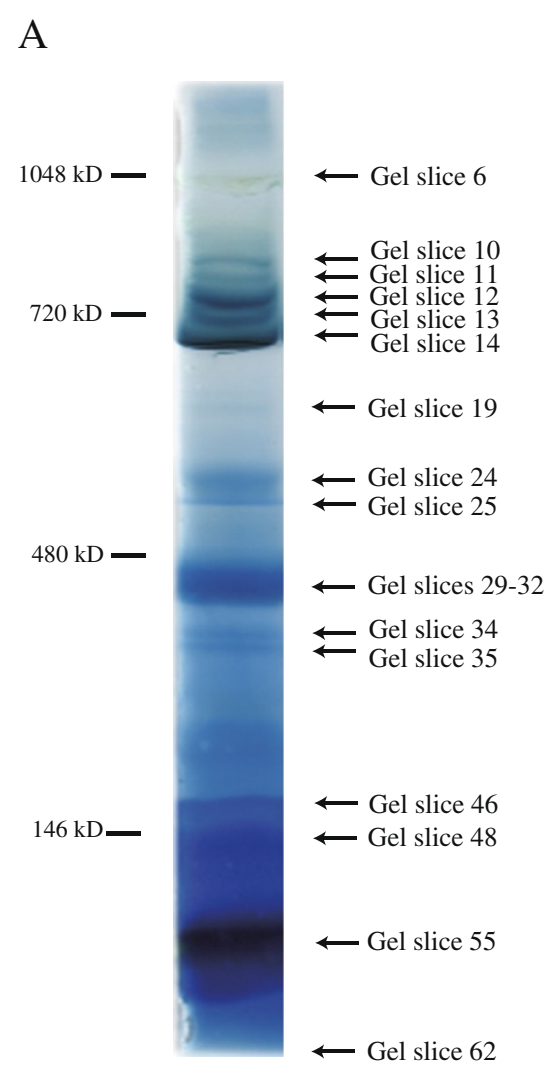

B

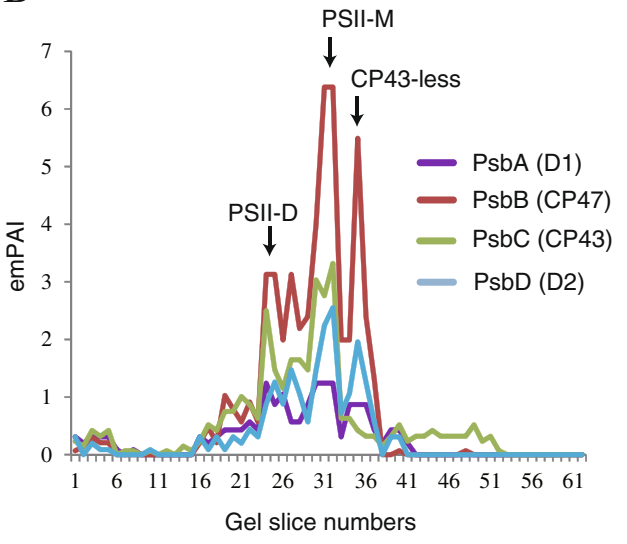

Sy 4 The application of the BN-PAGE methoin complexes from Synechocystis cells by BN-PAGE. Protein bands were visualized by CBB-staining (A). Protein migration profiles of PSIl core subunits in Synechocystis (B). Putative peaks corresponding to the PSII dimer (PSII-D), PSII monomer (PSII-M), and CP43-less PSII were shown.

DNase treatment was performed to remove the genomic DNA that was reported to significantly interfere with BN-PAGE using the whole cells of bacteria (Liang et al. 2009). The protein complexes were mildly solubilized with dodecyl maltoside and then separated by BN-PAGE (Figure 4). The BN gel lanes were cut horizontally from the top of the gel to just before the dye front, and the gel slices were then processed by in-gel digestion with trypsin and analyzed by LC-MS/MS. As a result, we identified 1,458 Synechocystis proteins, which were accounting for $41 \%$ of the total number of all the predicted gene models (Additional file 5: Table S3). According to the functional category by CyanoBase (http://genome.kazusa. or.jp/cyanobase), identified proteins were distributed all over functional categories (Additional file 6: Table S4). Notably, 554 proteins were classified into "Hypothetical" or "Unknown" (Additional file 6: Table S4).

We then generated the emPAI-based migration profiles for identified proteins based on the Mascot data from each gel (Additional file 7: Table S5). For examples, the peaks in their migration profiles corresponding to the putative PSII dimer (slices 24 and 25), the PSII monomer (slices 31 and 32), and the CP43-less PSII (the slice 35) were well correlated with the oligomeric states of PSII in Synechocystis according to the previous paper (Herranen et al. 2004) (Figure 4B). These data shows that the BN-PAGE coupled with LC-MS/MS was useful, even when whole cells were used.

\section{Building a Protein Co-migration Database for Photosynthetic Organisms (PCoM-DB)}

Because emPAI-based migration profiles provide us useful information regarding protein complexes, we built a web application called PCoM-DB (http://pcomdb.lowtem.hokudai. ac.jp/proteins/top) that shows and compares the migration profiles of the identified proteins in this study (Figure 5).

PCoM-DB is freely accessible through the web browsers. The emPAI-based migration profiles shown in PCoM-DB provide users the following information regarding identified proteins: 1) the number of putative protein complexes associated with a protein of interest; 2) the estimated molecular sizes of the protein complexes associated with the protein of interest; and 3) the relative accumulation levels of each protein complex associated with the protein of interest. In addition, users 


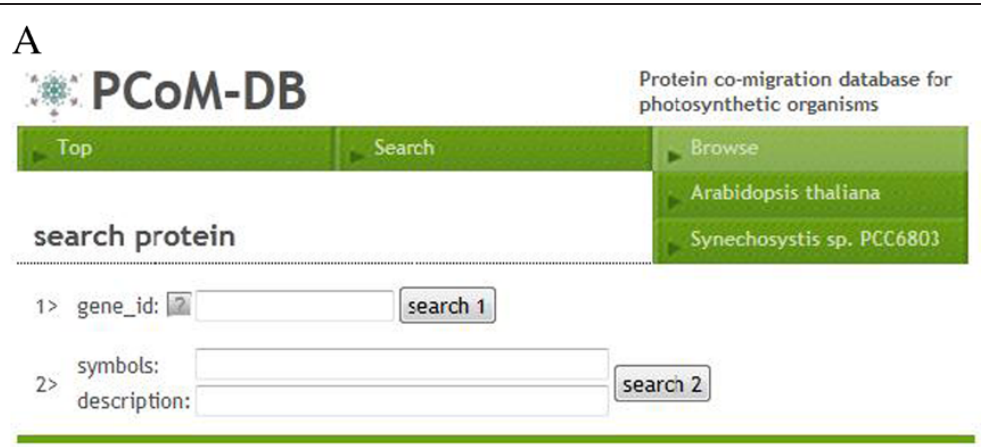

\section{B}

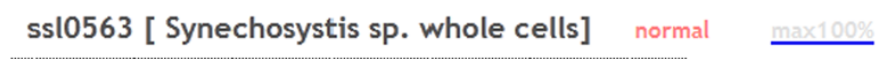

symbols : psac

description :photosystem I subunit VII

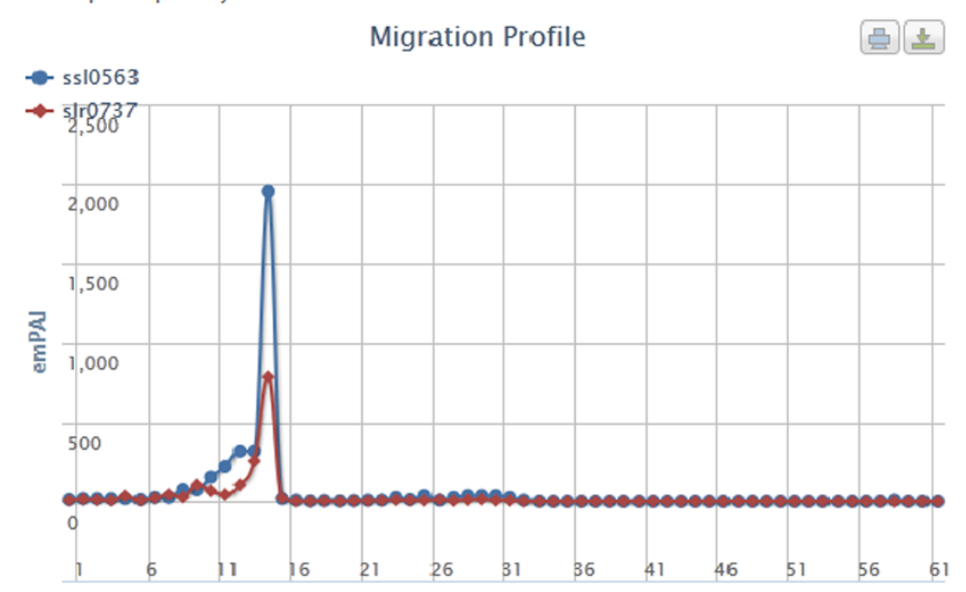

gel_slice_number

\begin{tabular}{|c|c|c|c|c|}
\hline gel_no & 6 & 13 & 28 & 48 \\
\hline $\mathrm{mw}$ & 1048 & 720 & 480 & 146 \\
\hline
\end{tabular}

reCharts AllCheck Clear point selected : gel_no_15

\begin{tabular}{|l|l|l|l|r|r|r|l|}
\hline ^gene_id & peak & emPAl & $\begin{array}{c}\text { Imax } \\
\text { emPAl(\%) }\end{array}$ & mw & symbols & \multicolumn{1}{c|}{ description } \\
\hline$\nabla$ & $\underline{\text { ssl0563 }}$ & P & 1952.99 & 100.0 & 9335 & psaC & $\begin{array}{l}\text { photosystem I subunit } \\
\text { VII }\end{array}$ \\
\hline$\square$ & $\underline{\text { slr0737 }}$ & P & 784.58 & 100.0 & 15691 & psaD & photosystem I subunit II
\end{tabular}

Figure 5 Browse and search interfaces in PCoM-DB (A), and comparing the migration profiles of Synechocystis PsaC and PsaD proteins using PCoM-DB (B). Users can easily find the protein migration profile of a protein of interest through browse and search interfaces $(\mathbf{A})$. Then, users also can find the potential interacting partners by finding the proteins which shared the peaks in the protein migration profiles using PCoM-DB (A). In addition, users can compare the overall protein migration profiles of potential interacting partners with a protein of interest. In this case, protein migration profiles of PsaC and PsaD were shown as an example (B). The migration profiles of a Synechocystis PsaC protein had a peak in the gel slice 15, corresponding to the PSI trimer. Only clicking the blue closed circle showing the PsaC emPAl value at the gel slice 15, users can find the proteins which were also detected in the gel slice 15 as potential interacting partners (and one of them was PsaD).

can find co-migrated proteins with a protein of interest, based on the assumption that the proteins whose peaks in their migration profile overlap within the same gel slice possibly interact with each other. PCoM-DB helps users to find the potential interacting partners with a protein of interest.

\section{Discussion}

In this study, we analyzed thylakoid and cyanobacterial protein complexes using the BN-PAGE coupled with LC-MS/MS. We found that the protein migration profiles of the PSII, PSI, cytochrome $b_{6} / f$, ATP synthase, and NDH subunits in Arabidopsis thylakoids were well 
correlated with their oligomeric states within the $\mathrm{BN}$ gel. Also, the protein migration profiles of the PSII core proteins in Synechocystis cells were similar with each other, and should be correlated with the oligomeric states of PSII in the previous report.

BN-PAGE coupled with LC-MS/MS is expected to be much more sensitive than 2D-BN/SDS-PAGE followed by MS analysis because the sensitivities of the CBB, silver, and fluorescent staining used for the visualization of protein spots are lower than the sensitivity of protein identification with LC-MS/MS. However, the likelihood of the MS-based protein detection varied greatly depending on the properties of each peptide. Especially, LC-MS/MS typically has difficulty in detecting very hydrophobic proteins. Therefore, thylakoid membrane protein complexes may not be suitable for comprehensive identification by LC-MS/MS. On the other hand, 1,458 proteins were identified in the whole cells of Synechocystis. This number is equivalent to $41 \%$ of all predicted gene model, even though some proteins may not always expressed in Synechocystis cells. According to the functional categories in CyanoBase, identified proteins in Synechocystis cells distributed all over functional categories and well contained the unknown proteins. We think that the low number of total proteins encoded in bacterial genome has a great advantage for high sensitivity in LC-MS /MS, because the protein identification probability by searching sequence databases using mass spectrometry data should be higher. Our data presented here showed that BN-PAGE coupled with LC-MS/MS method is expected to be suitable for the large-scale identification of protein complexes, especially in bacterial cells.

Finally, we built a web database called PCoM-DB to make our data publicly accessible online. PCoM-DB shows users the migration profiles of proteins of interest. The protein migration profiles provide important information regarding the protein complexes. First, the protein migration profiles display the number and the estimated sizes of the protein complexes associated with a protein of interest. Second, they also provide a list of the co-migrated proteins whose protein migration profile peaks were overlapped with those of the protein of interest, or whose migration profiles were similar to those of the protein of interest. The co-migrating proteins with a protein of interest are potential interacting partners with the protein of interest. Different sources of biological information, such as gene expression data, protein domain information data, and mutant phenotypes, can be used for the further selection of the potential interacting partners.

At present, our database contains the data presented here (Arabidopsis thylakoid membrane proteins and Synechocystis sp. PCC 6803 proteins). We plan to add data to PCoM-DB from other cyanobacteria in addition to additional compartments from Arabidopsis. The accumulation of the data in PCoM-DB should help researchers to find unknown protein complexes.

\section{Methods \\ Growth conditions}

The Columbia ecotype of Arabidopsis thaliana was used as the wild type in this study. The wild-type plants were grown in soil under constant illumination $(70 \mu \mathrm{mol}$ photon $\mathrm{m}^{-2} \mathrm{~s}^{-1}$ ) at $23^{\circ} \mathrm{C}$. The growth conditions of Synechocystis sp. PCC 6803 were described previously (Satoh et al. 2001).

\section{Isolation of Arabidopsis thylakoid membranes}

All of the following procedures were conducted at $4{ }^{\circ} \mathrm{C}$ unless otherwise stated. The thylakoid membranes were isolated from the leaves of 4-week-old plants. The thylakoid membranes were prepared essentially according to the methods described by Salvi et al. (Salvi et al. 2008). Briefly, the leaves were homogenized in isolation buffer containing $20 \mathrm{mM}$ Tricine- $\mathrm{NaOH}$ (pH 8.0), $0.45 \mathrm{M}$ sorbitol, $10 \mathrm{mM}$ EDTA-2Na, 1 mM NaHCO 3 , 0.1\% (w/v) BSA, 0.05\% (w/v) DTT, and $0.05 \%(\mathrm{w} / \mathrm{v})$ PVP. The homogenate was filtered through four layers of Miracloth and centrifuged at $1,000 \times g$ for $5 \mathrm{~min}$. The pellet was suspended in wash buffer containing $20 \mathrm{mM}$ MOPS-NaOH (pH 7.6), $0.33 \mathrm{M}$ sorbitol, $5 \mathrm{mM} \mathrm{MgCl}_{2}$, and $2.5 \mathrm{mM}$ EDTA-2Na. After a Percoll density gradient centrifugation, the intact chloroplasts were collected from the interface between $40 \%$ and $80 \%$ Percoll. The intact chloroplast suspension was washed twice and then osmotically ruptured in swelling buffer containing $10 \mathrm{mM}$ MOPS-NaOH ( $\mathrm{pH} 7.6$ ) and 4 $\mathrm{mM} \mathrm{MgCl}_{2}$. After a sucrose density gradient (2 M, $0.93 \mathrm{M}$ and $0.6 \mathrm{M}$ ) centrifugation at 95,000 $\times g$ in a RPS56T rotor (Hitachi, Tokyo, Japan) for $60 \mathrm{~min}$ at $4^{\circ} \mathrm{C}$, the purified thylakoid membranes were collected from the interface between $0.93 \mathrm{M}$ and $2 \mathrm{M}$ sucrose.

\section{BN-PAGE}

BN-PAGE was performed essentially according to the methods described by Wittig et al. (Wittig et al. 2006). The chlorophyll content was determined according to Porra et al. (Porra et al. 1989).

Thylakoid membranes purified from Arabidopsis were suspended at a concentration of $1 \mathrm{mg} / \mathrm{ml}$ chlorophyll $a$ and $b$ in ice-cold resuspension buffer containing $50 \mathrm{mM}$ imidazole- $\mathrm{HCl}$ ( $\mathrm{pH} 7.0$ ), 20\% glycerol, $5 \mathrm{mM}$ 6-aminocaproic acid, and $1 \mathrm{mM}$ EDTA-2Na. Cells of Synechocystis were harvested by centrifugation and homogenized using 0.1 $\mathrm{mm}$ glass beads in a vortex mixer in ice-cold resuspension buffer. The genomic DNA was removed using Benzonase Nuclease (Merck, Darmstadt, Germany) for $30 \mathrm{~min}$ on ice. The suspension ( $1 \mathrm{mg} / \mathrm{ml}$ chlorophyll) was solubilized with $1 \%(\mathrm{w} / \mathrm{v}) \mathrm{n}$-dodecyl- $\beta$-D-maltoside on ice for $5 \mathrm{~min}$. After centrifugation at $18,800 \times g$ for $10 \mathrm{~min}$ at $4^{\circ} \mathrm{C}$, the 
supernatants were supplemented with Coomassie Blue solution [5\% (w/v) Serva Blue G (Serva, Heidelberg, Germany), $500 \mathrm{mM} \mathrm{6-aminocaproic} \mathrm{acid,} \mathrm{and} 50 \mathrm{mM}$ Imidazole- $\mathrm{HCl}(\mathrm{pH}$ 7.0)] to a detergent/Coomassie ratio of $4 / 1(\mathrm{w} / \mathrm{w})$. The samples $(4 \mu \mathrm{g}$ chlorophyll equivalent) were separated in $4-13 \%$ polyacrylamide gradient gels at $4{ }^{\circ} \mathrm{C}$ for $14 \mathrm{~h}$ at $40 \mathrm{~V}$.

\section{Nano-LC/MS/MS for proteomics of Arabidopsis thylakoids and Synechocystis cells}

The BN-PAGE gel lanes were cut horizontally into approximately 60 slices at regular intervals (ca. $1 \mathrm{~mm}$ ) from the top of the gel to just before the dye front. All gel slices were treated by in-gel digestion with trypsin (Roche) according to Shevchenko et al. (Shevchenko et al. 1996).

LC-MS/MS analyses were performed using an LTQ iontrap MS (Thermo Fisher Scientific, Yokohama, Japan) coupled with an HPLC (AMR Inc., Tokyo, Japan) and a nano-spray electrospray ionization device (Michrom Bioresources Inc., CA, USA), essentially according to Kasahara et al. (2012) (Kasahara et al. 2012). The resulting peptides were loaded onto an L-column 2 ODS column packed with $\mathrm{C} 18(5 \mu \mathrm{m}, 12 \mathrm{~nm}$ pore size) (Chemicals Evaluation and Research Institute, Japan) and separated by a gradient using solvent $\mathrm{A}$ ( $2 \%$ acetonitrile in $0.1 \%$ formic acid) and solvent B (90\% acetonitrile in $0.1 \%$ formic acid). The gradient condition was 5\% B to $90 \%$ B and the flow rate was $1 \mu \mathrm{L} / \mathrm{min}$. The separated peptides were analyzed with an LTQ for MS/MS analysis. The ESI conditions for the LTQ instrument were set as follows: capillary temperature of $200^{\circ} \mathrm{C}$ and a spray voltage of $2.0 \mathrm{kV}$. The scan range was $\mathrm{m} / \mathrm{z} 450-1800$ for LTQ. The scan time for MS and MS/MS were $0.6 \mathrm{~s}$. The ion selection threshold, the minimum signal required for $\mathrm{MS} / \mathrm{MS}$, was set to 200 counts. MS/MS spectra were acquired in datadependent scan mode. After the full spectrum scan, one MS/MS spectrum of the single most intense peaks was also collected.

Protein identification was performed as follows. Raw files were converted to a Mascot generic file (mgf) and searched with Mascot version 2.2 (Matrix Science) into the TAIR9 database (The Arabidopsis Information Resource; http://www.arabidopsis.org; Total sequences, 33410; Total residues, 13434913) for Arabidopsis thylakoid proteins or searched with the Mascot version 2.3 into the protein data from CyanoBase (http://genome. kazusa.or.jp/cyanobase/; Total sequences, 3569; Total residues, 1137101) for Synechocystis proteins. The results were filtered with a significance threshold of $\mathrm{p}<0.05$ for peptides identifications and the MudPIT scoring algorism was used. Also, the following search parameters were used: 0.05 for ions score cut off, $1.2 \mathrm{Da}$ peptide tolerance; $\pm 0.8 \mathrm{Da}$ MS/MS tolerance; $1+, 2+$, or
$3+$ peptide charge; trypsin digestion with two missed cleavage allowed; carbamidomethyl modification of cysteines as a fixed modification; and oxidation of methionine as a variable modification. Furthermore, only the top scoring peptide match was used in this study and we accepted proteins identified by at least one unique peptide. False discovery rates (FDR) for peptide matches above the identity threshold were 4.54\% for Arabidopsis thylakoids and $2.83 \%$ for Synechocystis cells, respectively. The detailed Mascot data for identified peptides were shown in Additional file 8: Table S6 for Arabidopsis thylakoids and Additional file 9: Table S7 for Synechocystis cells.

\section{Conclusion}

Here, we applied the BN-PAGE followed by LC-MS/MS method for comprehensive identification of protein complexes into the thylakoid protein complexes of Arabidopsis and whole cell protein complexes of Synechocystis sp. PCC 6803. The emPAI-based protein migration profiles based on the data from the method were useful for finding protein complexes, especially for Synechocystis. The web database called PCoM-DB to make our data publicly accessible online has been constructed. PCoM-DB, which stores the analyzed data with a user-friendly interface, can help users to find novel protein complexes in Arabidopsis thylakoids and Synechocystis.

\section{Additional files}

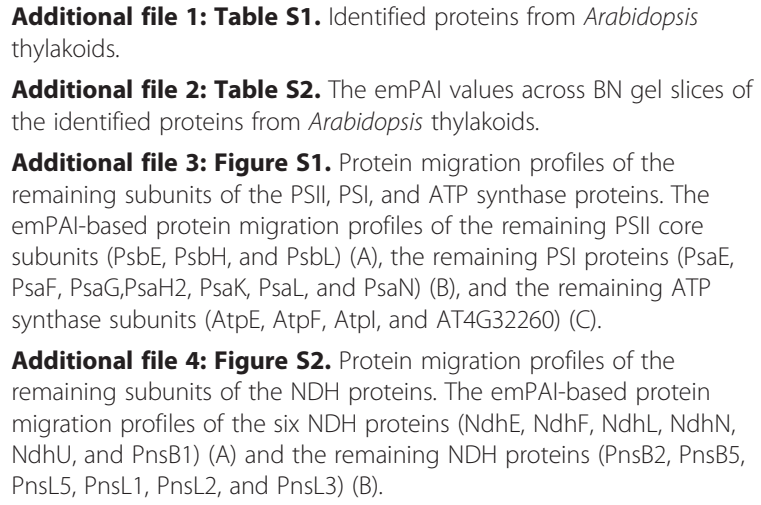

Additional file 3: Figure S1. Protein migration profiles of the remaining subunits of the PSII, PSI, and ATP synthase proteins. The emPAl-based protein migration profiles of the remaining PSII core subunits (PsbE, PsbH, and PsbL) (A), the remaining PSI proteins (PsaE, PsaF, PsaG,PsaH2, PsaK, PsaL, and PsaN) (B), and the remaining ATP synthase subunits (AtpE, AtpF, Atpl, and AT4G32260) (C).

Additional file 4: Figure S2. Protein migration profiles of the remaining subunits of the NDH proteins. The emPAl-based protein migration profiles of the six NDH proteins (NdhE, NdhF, NdhL, NdhN, NdhU, and PnsB1) (A) and the remaining NDH proteins (PnsB2, PnsB5, PnsL5, PnsL1, PnsL2, and PnsL3) (B).

Additional file 5: Table S3. Identified proteins from Synechocystis cells Additional file 6: Table S4. The emPAI values across BN gel slices of the identified proteins from Synechocystis cells.

Additional file 7: Table S5. Functional classification of identified Synechocystis proteins according to CyanoBase.

Additional file 8: Table S6. Mascot data for identified peptides from Arabidopsis thylakoids.

Additional file 9: Table S7. Mascot data for identified peptides from Synechocystis cells.

\section{Competing interest}

The authors declare that they have no competing interests. 


\section{Authors' contributions}

AT (Atsushi Takabayashi) contributed to the experimental design, data collection, data analysis, data interpretation, and drafted the manuscript. RK and MK carried out the Mass spectrometry studies. KK carried out the BNPAGE studies. HI carried out the preparation of whole cell proteins of Synechocystis. RT and AT (Ayumi Tanaka) participated the experimental design, data interpretation, and drafted the manuscript. All authors read and approved the final manuscript.

\section{Acknowledgments}

We are very grateful to Ms. Saeka Takabayashi for constructing a PCoM-DB. This work was financially supported by the Grants-in-Aids for Young Scientists (23770035 to Atsushi Takabayashi) and by Scientific Research (10197402 to R.T.) from the Japan Society for the Promotion of Science.

Received: 24 January 2013 Accepted: 25 March 2013

Published: 8 April 2013

\section{References}

Arabidopsis Interactome Mapping Consortium (2011) Evidence for Network Evolution in an Arabidopsis Interactome Map. Science 333(6042):601-607. doi:10.1126/science.1203877

Braun P, Aubourg S, Van Leene J, De Jaeger G, Lurin C (2013) Plant Protein Interactomes. Annu Rev Plant Biol. doi:10.1146/annurev-arplant-050312120140

Fandino AS, Rais I, Vollmer M, Elgass H, Schägger H, Karas M (2005) LC-nanosprayMS/MS analysis of hydrophobic proteins from membrane protein complexes isolated by blue-native electrophoresis. J Mass Spectrom: JMS 40(9):1223-1231. doi:10.1002/jms.903

Ferro M, Brugiere S, Salvi D, Seigneurin-Berny D, Court M, Moyet L, Ramus C, Miras S, Mellal M, Le Gall S, Kieffer-Jaquinod S, Bruley C, Garin J, Joyard J, Masselon C, Rolland N (2010) AT_CHLORO, a comprehensive chloroplast proteome database with subplastidial localization and curated information on envelope proteins. Mol Cell Proteomics 9(6):1063-1084. doi:10.1074/mcp.M900325-MCP200

Heide H, Bleier L, Steger M, Ackermann J, Drose S, Schwamb B, Zornig M, Reichert AS, Koch I, Wittig I, Brandt U (2012) Complexome profiling identifies TMEM126B as a component of the mitochondrial complex I assembly complex. Cell Metab 16(4):538-549. doi:10.1016/j.cmet.2012.08.009

Helbig AO, de Groot MJ, van Gestel RA, Mohammed S, de Hulster EA, Luttik MA Daran-Lapujade P, Pronk JT, Heck AJ, Slijper M (2009) A three-way proteomics strategy allows differential analysis of yeast mitochondrial membrane protein complexes under anaerobic and aerobic conditions. Proteomics 9(20):4787-4798. doi:10.1002/pmic.200800951

Herranen M, Battchikova N, Zhang P, Graf A, Sirpio S, Paakkarinen V, Aro EM (2004) Towards functional proteomics of membrane protein complexes in Synechocystis sp. PCC 6803. Plant Physiol 134(1):470-481. doi:10.1104/ pp.103.032326

Ifuku K, Endo T, Shikanai T, Aro EM (2011) Structure of the chloroplast NADH dehydrogenase-like complex: nomenclature for nuclear-encoded subunits. Plant Cell Physiol 52(9):1560-1568. doi:10.1093/pcp/pcr098

Ishihama Y, Oda Y, Tabata T, Sato T, Nagasu T, Rappsilber J, Mann M (2005) Exponentially modified protein abundance index (emPAl) for estimation of absolute protein amount in proteomics by the number of sequenced peptides per protein. Mol Cell Proteomics 4(9):1265-1272. doi:10.1074/mcp. M500061-MCP200

Kasahara Y, Morimoto H, Kuwano M, Kadoya R (2012) Genome-wide analytical approaches using semi-quantitative expression proteomics for aromatic hydrocarbon metabolism in Pseudomonas putida F1. J Microbiol Methods 91 (3):434-442. doi:10.1016/j.mimet.2012.09.017

Kim WY, Kang S, Kim BC, Oh J, Cho S, Bhak J, Choi JS (2008) SynechoNET: integrated protein-protein interaction database of a model cyanobacterium Synechocystis sp. PCC 9(1):20. doi:10.1186/1471-2105-9-S1-S20

Liang J, Niu Q, Xu X, Luo Y, Zhou X, Deng Z, Wang Z (2009) Effective elimination of nucleic acids from bacterial protein samples for optimized blue native polyacrylamide gel electrophoresis. Electrophoresis 30(14):2454-2459. doi:10.1002/elps.200900026

Lu P, Vogel C, Wang R, Yao X, Marcotte EM (2007) Absolute protein expression profiling estimates the relative contributions of transcriptional and translational regulation. Nat Biotechnol 25(1):117-124. doi:10.1038/nbt1270
Neilson JA, Durnford DG (2010) Structural and functional diversification of the light-harvesting complexes in photosynthetic eukaryotes. Photosynth Res 106(1-2):57-71. doi:10.1007/s11120-010-9576-2

Porra RJ, Thompson WA, Kriedemann PE (1989) Determination of accurate extinction coefficients and simultaneous-equations for assaying chlorophyll-a and chlorophyll-b extracted with 4 different solvents - verification of the concentration of chlorophyll standards by atomic-absorption spectroscopy. Biochim Biophys Acta 975(3):384-394

Remmerie N, De Vijlder T, Valkenborg D, Laukens K, Smets K, Vreeken J, Mertens I, Carpentier SC, Panis B, De Jaeger G, Blust R, Prinsen E, Witters E (2011) Unraveling tobacco BY-2 protein complexes with BN PAGE/LC-MS/MS and clustering methods. J Proteomics 74(8):1201-1217. doi:10.1016/j.jprot.2011.03.023

Salvi D, Rolland N, Joyard J, Ferro M (2008) Purification and proteomic analysis of chloroplasts and their sub-organellar compartments. Meth Mol Biol 432:19-36. doi:10.1007/978-1-59745-028-7_2

Satoh S, Ikeuchi M, Mimuro M, Tanaka A (2001) Chlorophyll b expressed in cyanobacteria functions as a light-harvesting antenna in photosystem I through flexibility of the proteins. J Biol Chem 276(6):4293-4297. doi:10.1074/ jbc.M008238200

Schägger H, von Jagow G (1991) Blue native electrophoresis for isolation of membrane protein complexes in enzymatically active form. Anal Biochem 199(2):223-231

Schulze WX, Usadel B (2010) Quantitation in mass-spectrometry-based proteomics. Annu Rev Plant Biol 61:491-516. doi:10.1146/annurev-arplant042809-112132

Shevchenko A, Wilm M, Vorm O, Mann M (1996) Mass spectrometric sequencing of proteins silver-stained polyacrylamide gels. Anal Chem 68(5):850-858

Takabayashi A, Kurihara K, Kuwano M, Kasahara Y, Tanaka R, Tanaka A (2011) The oligomeric states of the photosystems and the light-harvesting complexes in the Chl b-less mutant. Plant Cell Physiol 52(12):2103-2114. doi:10.1093/pcp/pcr138

Wessels HJ, Vogel RO, van den Heuvel L, Smeitink JA, Rodenburg RJ, Nijtmans LG, Farhoud MH (2009) LC-MS/MS as an alternative for SDS-PAGE in blue native analysis of protein complexes. Proteomics 9(17):4221-4228. doi:10.1002/ pmic.200900157

Wiethaus J, Busch AW, Dammeyer T, Frankenberg-Dinkel N (2010) Phycobiliproteins in Prochlorococcus marinus: biosynthesis of pigments and their assembly into proteins. Eur J Cell Biol 89(12):1005-1010. doi:10.1016/j. ejcb.2010.06.017

Wittig I, Schägger H (2008) Features and applications of blue-native and clearnative electrophoresis. Proteomics 8(19):3974-3990. doi:10.1002/ pmic.200800017

Wittig I, Braun HP, Schägger H (2006) Blue native PAGE. Nat Protoc 1(1):418-428 doi:10.1038/nprot.2006.62

doi:10.1186/2193-1801-2-148

Cite this article as: Takabayashi et al:: Protein co-migration database (PCoM -DB) for Arabidopsis thylakoids and Synechocystis cells. SpringerPlus 2013 2:148

\section{Submit your manuscript to a SpringerOpen ${ }^{\circ}$ journal and benefit from:}

- Convenient online submission

- Rigorous peer review

- Immediate publication on acceptance

- Open access: articles freely available online

- High visibility within the field

- Retaining the copyright to your article

Submit your next manuscript at $>$ springeropen.com 